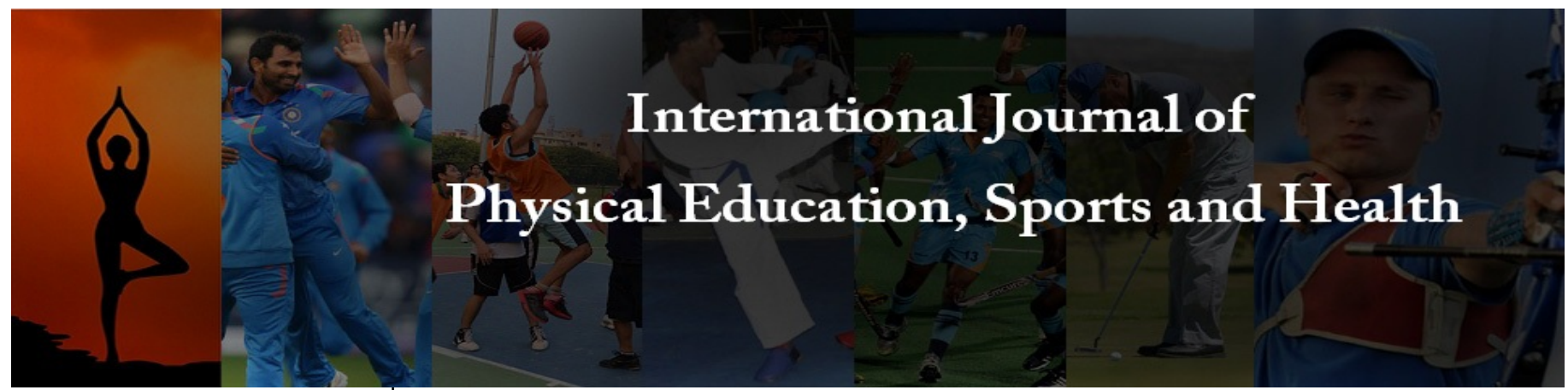

P-ISSN: 2394-1685

E-ISSN: 2394-1693

Impact Factor (ISRA): 5.38

IJPESH 2021; 8(4): 332-337

(C) 2021 IJPESH

www.kheljournal.com

Received: 07-05-2021

Accepted: 09-06-2021

Dr. Muhammed Najeeb K

Assistant Professor, Department of Physical Education, Amal College of Advanced Studies, Myladi, Nilambur, Malappuram, Kerala, India

Corresponding Author: Dr. Muhammed Najeeb K Assistant Professor, Department of Physical Education, Amal College of Advanced Studies, Myladi, Nilambur, Malappuram, Kerala, India

\section{Effect of Kalaripayattu on selected physical and psychological variables of school children}

\author{
Dr. Muhammed Najeeb K
}

DOI: https://doi.org/10.22271/kheljournal.2021.v8.i4f.2201

\section{Abstract}

The purpose of the study is to find out the effect of Kalaripayattu on selected physical and psychological variables of school children. Forty school boys $(\mathrm{N}=40)$ were selected from Kanuur district of Kerala for the study. The total subjects were divided in to two group namely experimental group $\left(\mathrm{n}_{\mathrm{m}}=20\right)$ and control group $\left(\mathrm{n}_{\mathrm{c}}=20\right)$. The subjects had no training background and their ages were between $10-14$ years. The experimental group participated Kalaripayattu training program 6 days in a week for a period of 2 months. The following Physical variables such as explosive power and flexibility and the following psychological variables namely; aggression and confidence level were selected for the purpose of the study. Explosive power of the subjects was collected by using Standing broad jump test and Flexibility collected by using sit and reach test whereas Aggression of the subjects was collected by using the questionnaire developed by Tiware and Chauhan and the confidence level data was collected from the subjects by using the questionnaire developed by Philippa Davied "how do you respond". Shapiro - Wilk test and Box Plot were used to determine whether the collected data was normally distributed or not. Then, with the consultation of statistical expert, we chose the appropriate statistics for the effect of Kalaripayattu training on physical and psychological variables of school children. The calculated numerical results were interpreted meaningfully. In all cases, the criterion for statistical significance was set at 0.05 level of confidence $(P<0.05)$. All the data were analysed with the help of SPSS version 23.

Keywords: Kalaripayattu, physical, psychological variables, school children

\section{Introduction}

Martial arts are codified systems and traditions of combat practices, which are practiced for a number of reasons: as self-defence, military and law implementation, mental and spiritual development as well as entertainment and the preservation of a nation's intangible cultural heritage. Martial arts are practiced for a variety of different reasons including self-defense, physical fitness, entertainment and competition. Some even consider martial arts as a way of achieving spiritual growth. Here is a list of the top martial arts today, not in any particular order (Eva Dixon, April 16, 2014).

Martial arts attempt to strike coordination between the mind and body along with training effective methods of self-defence. Martial arts for children encourage the all-round development of a child by developing the intellectual abilities, spiritual strength of the child along with his physical capabilities. According to experts, six years is an appropriate age for teaching martial arts. By that age, a child should have ideally developed enough muscle strength and control to apply the various techniques of martial arts like punches, kicks and so on. The focus is more on developing skills like hand-eye coordination and improving the attention span. (Aliya Khan, 2018) ${ }^{[3]}$.

Kalaripayattu, also known as Kalari, is an Indian martial art and fighting style that originated in Kerala, Gods own country. Kalaripayattu has different techniques like meipayattu (physical body exercise), vadipayattu (fight using sticks), valpayattu (fight using swords) and verumkaiprayoga (bare hand exercise). Kalari treatment is a part of the program. In Kalaripayattu, the first exercise is body exercise. Oil massage is an essential part of body exercise and this is one of the unique characteristics of Kalaripayauttu. The body is well oiled to fine tune the body for the next step that is payattu (fight). With oral instruction, physical Body movements are practiced. In meipayattu, there are 8 to 16 payattu (fight) during different

$$
\sim 332 \sim
$$


stages. Meipayattu is followed by kolpayattu in which sticks of different lengths are used.

This involves defensive and offensive techniques with oral instructions.

In Meipayattu (physical body exercise), Wearing Katcha or loin cloth and covering the body with oil, the participants after prostrating before the deities and the Gurukkal begins his initial training with Meithari system, which forms the foundation for the entire range of Kalaripayattu training. By practicing meipayattu, every possible form of stretching, turning and twisting of the body is mastered. Participants are educated not to sleep during the day time and are advised to sleep well at night. It is important that the students understand that Kalari is not only a means of self-defence but also a means to becoming determined and self-disciplined.

\section{Objectives of the study}

The purpose of the study is to determine the effect of Kalaripayattu on selected physical and psychological variables of school children.

\section{Significance of the Study}

1. To determine the effect of Kalaripayattu training on aggression level and self-confidence of school children.

2. To find out the effect of Kalaripayattu training on explosive power and flexibility of school children.

\section{Review of Related Literature}

Rajith, T.R and Dr. A. Mahaboobjan (2016) ${ }^{[2]}$ conducted a similar study and the purpose of the study was to determine the effect of Kalaripayattu training programme on selected biochemical among intercollegiate basketball players. To achieve the purpose of the study, twenty-four intercollegiate basketball players were selected from the Bharathidasan University, Tiruchirapalli, Tamilnadu. The subjects were randomly selected and their age ranged from 20 -25 years. The selected groups were divided two groups, experimental and control group. The experimental group consisted of twelve basketball players and they underwent the medium of Kalaripayattu's selected skill training. Twelve Basketball players were not given any skill training, and they acted as control group. The duration of the training period was restricted to eight weeks and the session for alternative days in a week. Kalaripayattu training is considered as independent variables. The Biochemical variables (low density lipoprotein, total cholesterol and fasting blood sugar were measured in medical laboratory) were known as dependent variables. The statistical technique covariance ANCOVA was used to analyze the pre-test and post-test data of experimental group and control group. The results showed that the Kalarpayattu training group had improved the level of all the selected biochemical variables as compared to the control group.

\section{Methods}

\section{Selection of subjects}

Forty school boys $(\mathrm{N}=40)$ were selected from Kanuur district of Kerala for the study. The total subject was divided into two group namely experimental group $\left(\mathrm{n}_{\mathrm{m}}=20\right)$ and control group $\left(n_{c}=20\right)$. The subjects had no training background and their age ranged between 10 - 14 years.

The experimental group participated in meipayattu (physical body exercise) from Kalaripayattu training program for 6 days in a week for a period of 2 months. Meipayattu training refers to physical exercises that use the body, including specific exercises for legs, hips, hands and the upper portion of the body. These movements are all designed to help one achieve maximum physical fitness and body co-ordination. The control group did not participate in any sort of Kalaripayattu training programme during the same period.

\section{Selection of variables}

Independent variable

Kalaripayattu (Meipayauttu) - It constitutes the initial training system of Kalarippayattu. The term mei means body and payattu means training. Meipayauttu is considered to be the significant training part of the entire structure of the training. It combines unique body movements based on the integrity of the spine and the development of grip and postures of the body. This physical practice is very essential for the next stages of the training. Various stances and poses included in Meipayattu bear some resemblance with the postures of the wild animals such as lion, cat, boar, snake, cock etc.

\section{Dependent variable}

a) Physical variables - explosive power and flexibility

b) Psychological variables - Aggression and Confidence level

\section{Selection of Test Items}

Motor fitness variables

a) Explosive power - Standing broad jump test

b) Flexibility - sit and reach test

\section{Psychological variables}

a) Aggression - To measure the data on aggression level was collected from the subject by using the questionnaire developed by Tiware and Chauhan.

b) Confidence level - The confidence level data was collected from the subjects by using the questionnaire developed by Philippa Davied "how do you respond”.

\section{Statistical Techniques}

Shapiro - Wilk test and Box Plot were used to determine whether the collected data was normally distributed or not. Later, with the consultation of statistical expert, we chose appropriate statistics for determining the effect of Kalaripayattu training on physical and psychological variables of school children. The calculated numerical results were interpreted meaningfully. In all cases, the criterion for statistical significance was set at 0.05 level of confidence $(P<0.05)$. All the data were analyzed with the help of SPSS version 23.

\section{Analysis}

\section{Physical variables}

Shapiro - Wilk test and Box Plot were used to determine whether the collected data was normally distributed or not. 
Table 1: Normality test of physical variables

\begin{tabular}{|c|c|c|c|c|c|}
\hline \multirow{2}{*}{ Variable } & \multirow{2}{*}{ Group } & \multirow{2}{*}{ Test } & \multicolumn{3}{|c|}{ Shapiro-Wilk } \\
\cline { 3 - 6 } & & & Statistic & df & Sig. \\
\hline \multirow{3}{*}{ Explosive power } & \multirow{2}{*}{ Experimental } & Pre & 0.96 & 20.00 & 0.63 \\
\cline { 3 - 6 } & & Post & $0.83^{*}$ & 20.00 & 0.00 \\
\cline { 3 - 6 } & \multirow{2}{*}{ Control } & Pre & 0.94 & 20.00 & 0.29 \\
\cline { 3 - 6 } & & Post & 0.94 & 20.00 & 0.23 \\
\hline \multirow{3}{*}{ Flexibility } & \multirow{2}{*}{ Experimental } & Pre & 0.91 & 20.00 & 0.07 \\
\cline { 3 - 6 } & & Post & 0.95 & 20.00 & 0.39 \\
\cline { 3 - 6 } & \multirow{2}{*}{ Control } & Pre & 0.91 & 20.00 & 0.05 \\
\cline { 3 - 7 } & & Post & $0.86^{*}$ & 20.00 & 0.01 \\
\hline
\end{tabular}

\section{* Significant at the 0.05 level of confidence}

The above table indicates that, in the case of explosive power, $P$ value (0.63) of pre test of Experimental group is higher than the chosen alpha level (0.05 level), then the null hypothesis was accepted, but in the case of post test; $\mathrm{P}$ value $(0.00)$ control group is lesser than the chosen alpha level $(0.05$ level), then the null hypothesis was rejected. But in the case of control group; p values ( 0.29 and 0.23$)$ of pre and post test are higher than the chosen alpha level (0.05 level), then the null hypothesis is accepted. The evidence showed that the data tested were not normally distributed.
In the case of Flexibility, $\mathrm{P}$ values ( 0.07 and 0.39 ) of pre and post test Experimental group are higher than the chosen alpha level (0.05 level), then the null hypothesis is accepted. P value (0.05) of pre test of control group is equal to the chosen alpha level (0.05 level), then the null hypothesis was accepted, whereas, in the case of post test; $P$ value $(0.01)$ of control group is lesser than the chosen alpha level (0.05 level), then the null hypothesis was rejected. The evidence showed that the data tested were not normally distributed.
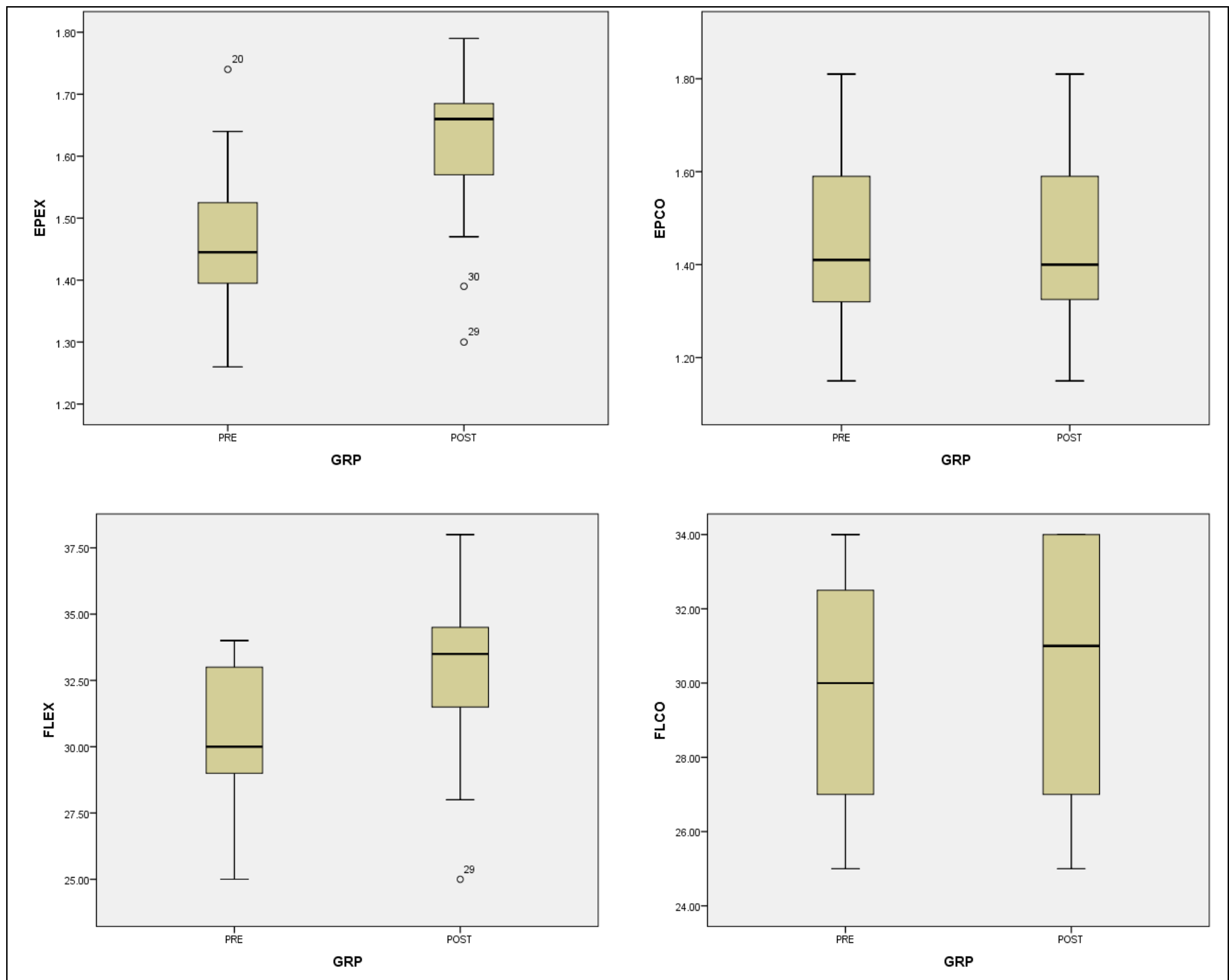

Fig 1: Box plots for physical variables

2. Psychological variables

Shapiro - Wilk test and Box Plot were used to determine whether the collected data was normally distributed or not. 
Table 2: Normality test of Psychological variables

\begin{tabular}{|c|c|c|c|c|c|}
\hline \multirow{2}{*}{ Variable } & \multirow{2}{*}{ Group } & \multirow{2}{*}{ Test } & \multicolumn{3}{|c|}{ Shapiro-Wilk } \\
\cline { 3 - 6 } & & & Statistic & df & Sig. \\
\hline \multirow{3}{*}{ Aggression } & \multirow{2}{*}{ Experimental } & Pre & 0.95 & 20.00 & 0.43 \\
\cline { 3 - 6 } & & Post & 0.92 & 20.00 & 0.09 \\
\cline { 3 - 6 } & \multirow{2}{*}{ Control } & Pre & $0.89^{*}$ & 20.00 & 0.02 \\
\cline { 3 - 6 } & & Post & $0.84^{*}$ & 20.00 & 0.00 \\
\hline \multirow{2}{*}{ Confidence Level } & \multirow{2}{*}{ Experimental } & Pre & $0.76^{*}$ & 20.00 & 0.00 \\
\cline { 3 - 6 } & & Post & $0.84^{*}$ & 20.00 & 0.00 \\
\cline { 3 - 6 } & \multirow{2}{*}{ Control } & Pre & 0.91 & 20.00 & 0.05 \\
\cline { 3 - 6 } & & Post & $0.89^{*}$ & 20.00 & 0.02 \\
\hline
\end{tabular}

* Significant at the 0.05 level of confidence

In the case of aggression, p values (0.43 and 0.09) of pre and post test of Experimental group are higher than the chosen alpha level (0.05 level), then the null hypothesis was accepted, but in control group, p values (0.02 and 0.00) of pre and post test are lesser than the chosen alpha level $(0.05$ level), then the null hypothesis is rejected. The evidence treated that the data tested were not normally distributed.

In the table indicated that, in the case of confidence level, p values (0.00 and 0.00$)$ of pre and post test of Experimental group are lesser than the chosen alpha level (0.05 level), then the null hypothesis is rejected. P value (0.05) of pre test of control group is higher than the chosen alpha level (0.05 level), then the null hypothesis was accepted, but in the case of post test; $\mathrm{P}$ value (0.02) control group is lesser than the chosen alpha level (0.05 level), then the null hypothesis was rejected. The evidence showed that the data tested were not normally distributed.

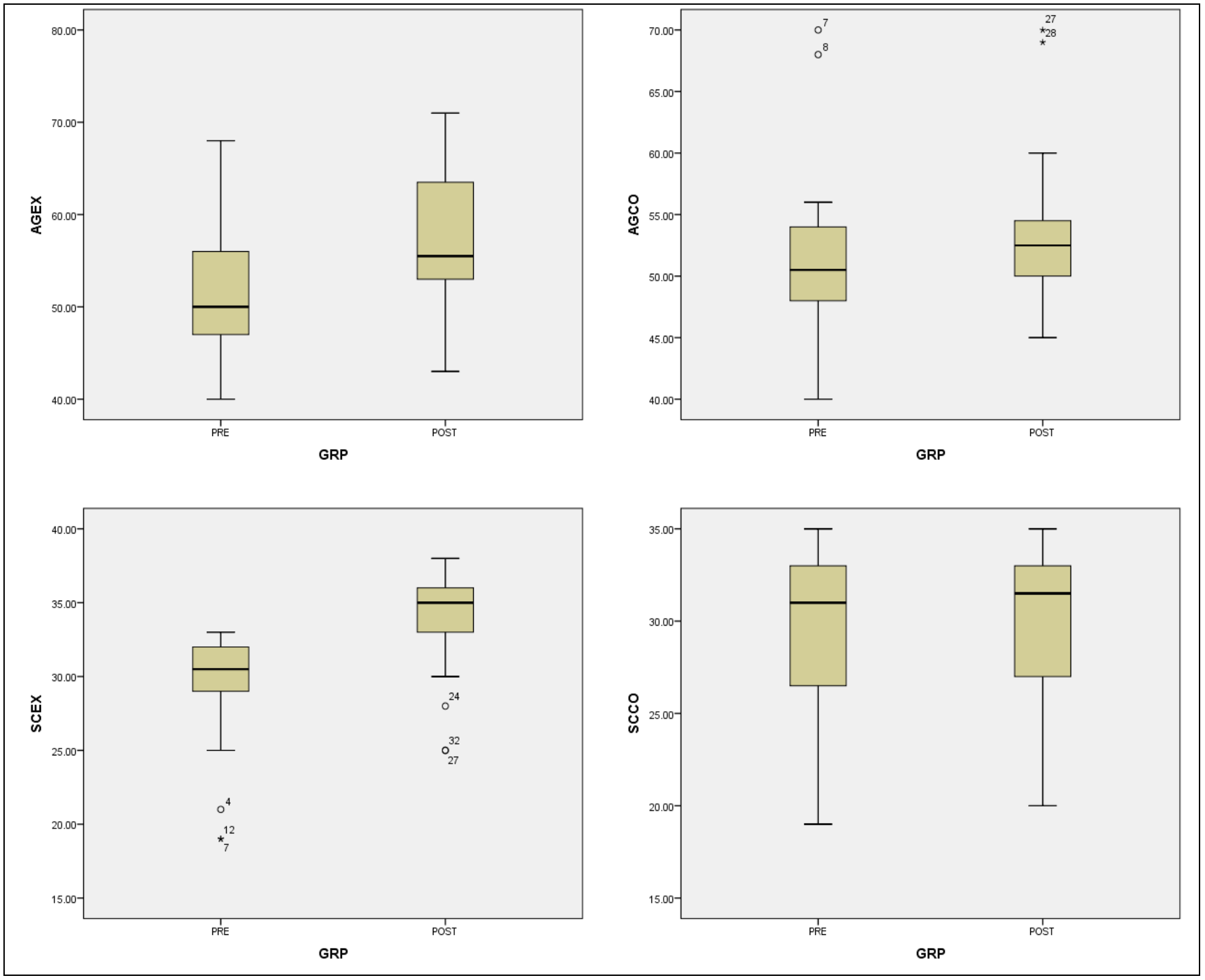

Fig 2: Box plots for Psychological variables

The evidences prove that, with the consultation of statistical expert, in the both physical variables (explosive power) and psychological variables (aggression and confidence level), the data were not normally distributed and Wilcoxon Signed Ranks Test (Non parametric test) was used for pair wise comparison. 


\section{Pair wise comparison}

1. Physical variables

Table 3: Comparison of physical variables

\begin{tabular}{|c|c|c|c|c|c|c|c|}
\hline Variable & Group & Rank & $\mathbf{N}$ & Mean Rank & Sum of Ranks & $\mathbf{Z}$ & Asymp. Sig. (2-tailed) \\
\hline \multirow{6}{*}{ Explosive power } & \multirow{3}{*}{ Experimental } & Negative Ranks & 0 & 0.00 & 0.00 & \multirow{3}{*}{$-3.92 *$} & \multirow{3}{*}{0.00} \\
\hline & & Positive Ranks & 20 & 10.50 & 210.00 & & \\
\hline & & Ties & 0 & & & & \\
\hline & \multirow{3}{*}{ Control } & Negative Ranks & 3 & 2.67 & 8.00 & \multirow{3}{*}{-1.13} & \multirow{3}{*}{0.27} \\
\hline & & Positive Ranks & 1 & 2.00 & 2.00 & & \\
\hline & & Ties & 16 & & & & \\
\hline \multirow{6}{*}{ Flexibility } & \multirow{3}{*}{ Experimental } & Negative Ranks & 5 & 7.80 & 39.00 & \multirow{3}{*}{$-2.26^{*}$} & \multirow{3}{*}{0.02} \\
\hline & & Positive Ranks & 14 & 10.79 & 151.00 & & \\
\hline & & Ties & 1 & & & & \\
\hline & \multirow{3}{*}{ Control } & Negative Ranks & 7 & 10.57 & 74.00 & \multirow{3}{*}{-0.50} & \multirow{3}{*}{0.62} \\
\hline & & Positive Ranks & 11 & 8.82 & 97.00 & & \\
\hline & & Ties & 2 & & & & \\
\hline
\end{tabular}

* Significant at the 0.05 level of Significance

From this table 4, it can be concluded that the explosive power between pre and post test of Experimental training group was statistically significant, since $\mathrm{p}$ value $(Z=-3.92, p$ $=0.00)$ was lesser than the chosen alpha level (0.05 level). Thus it can be concluded that the explosive power of the subjects was improved by Kalaripayattu training. There was no significant difference between pre and post test score of explosive power in the control group, since $\mathrm{p}$ value $(Z=$ $1.13, p=0.27)$ was higher than the chosen alpha level $(0.05$ level). Thus it can be concluded that in the case of explosive power, the control group was similar in nature.

In the case of Flexibility, $Z$ score $(Z=-2.26)$ between pre and post test of Experimental training group was statistically significant, since $\mathrm{p}$ value $(p=0.02)$ was lesser than the chosen alpha level (0.05 level). Thus it can be concluded that explosive power of the subjects improved by Kalaripayattu training. There was no significant difference between pre and post test score of explosive power in the control group, since $p$ value $(Z=-0.50, p=0.62)$ was higher than the chosen alpha level ( 0.05 level). Thus it can be concluded that in the case of flexibility, the control group was similar in nature.

\section{Psychological variables}

Table 4: Comparison of Psychological variables

\begin{tabular}{|c|c|c|c|c|c|c|c|}
\hline Variable & Group & Rank & $\mathbf{N}$ & Mean Rank & Sum of Ranks & $\mathbf{Z}$ & Asymp. Sig. (2-tailed) \\
\hline \multirow{6}{*}{ Aggression } & \multirow{3}{*}{ Experimental } & Negative Ranks & 19 & 11.00 & 209.00 & \multirow{3}{*}{$-3.89 *$} & \multirow{3}{*}{0.00} \\
\hline & & Positive Ranks & 1 & 1.00 & 1.00 & & \\
\hline & & Ties & 0 & & & & \\
\hline & \multirow{3}{*}{ Control } & Negative Ranks & 0 & 0.00 & 0.00 & \multirow{3}{*}{$-1.73 *$} & \multirow{3}{*}{0.08} \\
\hline & & Positive Ranks & 3 & 2.00 & 6.00 & & \\
\hline & & Ties & 17 & & & & \\
\hline \multirow{6}{*}{ Confidence Level } & \multirow{3}{*}{ Experimental } & Negative Ranks & 1 & 20.00 & 20.00 & \multirow{3}{*}{$-3.19 *$} & \multirow{3}{*}{0.00} \\
\hline & & Positive Ranks & 19 & 10.00 & 190.00 & & \\
\hline & & Ties & 0 & & & & \\
\hline & \multirow{3}{*}{ Control } & Negative Ranks & 2 & 3.00 & 6.00 & \multirow{3}{*}{$-1.00 *$} & \multirow{3}{*}{0.32} \\
\hline & & Positive Ranks & 4 & 3.75 & 15.00 & & \\
\hline & & Ties & 14 & & & & \\
\hline
\end{tabular}

* Significant at the 0.05 level of Significance

Table 3 reveals that, the aggression level between pre and post test of Experimental training group was statistically significant, since $\mathrm{p}$ value $(Z=-3.89, p=0.00)$ was lesser than the chosen alpha level ( 0.05 level). Thus it can be concluded that aggression level of the subjects was controlled by Kalaripayattu training. There was no significant difference between pre and post test score of aggression in the control group, since $p$ value $(Z=-1.73, p=0.08)$ was higher than the chosen alpha level ( 0.05 level). Thus it can be concluded that in the case of aggression level, the control group was similar in nature.

In the case of confidence level, $\mathrm{z}$ score $(Z=-3.89)$ between pre and post test of Experimental training group was statistically significant, since $\mathrm{p}$ value $(p=0.00)$ was lesser than the chosen alpha level (0.05 level). Thus it can be concluded that confidence level of the subjects increased due to Kalaripayattu training. There was no significant difference between pre and post test score of confidence level in the control group, since $p$ value $(Z=-1.00, p=0.32)$ was higher than the chosen alpha level (0.05 level). Thus it can be concluded that in the case of confidence level, the control group was similar in nature.

\section{Findings \\ 1 Physical variables}

Explosive leg power: In martial arts, having explosive power plays a huge role when it comes to delivering that gamechanging strike or submission. So any kind of martial arts can develop good explosive power. In the Kalaripayattu training, it involves many body movements, split jumps, rotator movements. All of these help develop explosiveness. The result of the study revealed that explosive power of the boys improved due to two months of Meipayattu training from Kalaripayattu martial art. 
Flexibility: In Kalaripayattu, we usually desire kinetic flexibility, also called- dynamic flexibility, the ability to move our muscles to bring a limb through its entire range of motion. By incorporating stretching exercises into the Kalaripayattu program, it can improve the range of motion of a joint or a series of joints, reduce joint pain, decrease risk of injury and improve ease of movement. The better flexibility of the Kalaripayattu practitioners may be due to their use of oil application before starting the payattu. Maipayattu movement with oil massage is a vital factor in improving the range of movement in selected school children.

\section{Psychological variables}

Aggression: Martial arts educate humility because learning one can be a humbling process. Martial arts could help reduce aggressive behaviors in youth, according to psychology researchers from Bar-Ilan University and UCLA. The result of the study shows that Kalaripayattu training reduced aggressiveness of the selected subjects. Kalaripayattu appear to be a useful intervention in reducing aggressive behavior.

In this study, the selected boys had no previous experience in any kind of martial arts practice. So, two months of Kalaripayattu training affected them both physically and mentally. Basically in our general life aggression is considered as a harmful negative emotion. So, regular Kalaripayattu practice can control aggression. However, many of the competitive martial arts practitioners might consider aggression as helpful negative emotion.

Confidence level: Martial arts can enhance the level of confidence to overcome any challenges that may encounter in our life. Martial arts not only makes one physically fit but also boosts our muscular strength. It also increases the level of self-esteem in public and other social places.

Through Kalaripayattu, we can learn to control our negative desires, get more concentration and focus on mastering more difficult skills. This comes from the discipline that students build by following instructions. The result of the study shows that Kalaripayattu training improved the level of confidence of the selected boys.

\section{Conclusions}

On the basis of the findings of the study, the following conclusions were drawn;

1. Significant difference in physical variables such as explosive power and flexibility, as a result of 2 months of Kalaripayattu programme for school children.

2. Significant difference in psychological variables namely aggression and confidence, as a result of 2 months of Kalaripayattu programme for school children.

\section{Recommendations}

1. The result of the study recommends that Kalaripayattu may be included in the school curriculum.

2. The findings of the present study can be used by Gurukals, teachers, Trainers and parents for assessing physical and psychological level of their children.

\section{References}

1. Deleury, Guy. India: The Rebel Continent. Macmillan. ISBN 1403924880, Martial art, Definition, History, Types, \& Facts. Encyclopedia Britannica 2005,89p. Retrieved 2020-01-13.

2. Rajith TR, Dr. A Mahaboobjan. Department of Physical
Education, Bharathidasan University, Tiruchirappalli, Tamilnadu, India. International Journal of Recent Research and Applied Studies 2016;3(11):59.

3. Khan, Aliya 2018, https://parenting.firstcry.com/art icles/martial-arts-for-childrens-benefits-and-more, retrieved on 20-04-2020

4. https://betterkidsinstitute.com/

5. https://villagefitnesssystems.com/ 